\title{
LEPTOQUARK QUANTUM NUMBERS
}

Revised September 2005 by M. Tanabashi (Tohoku University).

Leptoquarks are particles carrying both baryon number $(B)$ and lepton number $(L)$. They are expected to exist in various extensions of the Standard Model (SM). The possible quantum numbers of leptoquark states can be restricted by assuming that their direct interactions with the ordinary SM fermions are dimensionless and invariant under the SM gauge group. Table 1 shows the list of all possible quantum numbers with this assumption [1]. The columns of $\mathrm{SU}(3)_{C}, \mathrm{SU}(2)_{W}$, and $\mathrm{U}(1)_{Y}$ in Table 1 indicate the QCD representation, the weak isospin representation, and the weak hypercharge, respectively. The spin of a leptoquark state is taken to be 1 (vector leptoquark) or 0 (scalar leptoquark).

Table 1: Possible leptoquarks and their quantum numbers.

\begin{tabular}{cccccc}
\hline \hline Spin & $3 B+L$ & $\mathrm{SU}(3)_{c}$ & $\mathrm{SU}(2)_{W}$ & $\mathrm{U}(1)_{Y}$ & Allowed coupling \\
\hline 0 & -2 & $\overline{3}$ & 1 & $1 / 3$ & $\bar{q}_{L}^{c} \ell_{L}$ or $\bar{u}_{R}^{c} e_{R}$ \\
0 & -2 & $\overline{3}$ & 1 & $4 / 3$ & $\bar{d}_{R}^{c} e_{R}$ \\
0 & -2 & $\overline{3}$ & 3 & $1 / 3$ & $\bar{q}_{L}^{c} \ell_{L}$ \\
1 & -2 & $\overline{3}$ & 2 & $5 / 6$ & $\bar{q}_{L}^{c} \gamma^{\mu} e_{R}$ or $\bar{d}_{R}^{c} \gamma^{\mu} \ell_{L}$ \\
1 & -2 & $\overline{3}$ & 2 & $-1 / 6$ & $\bar{u}_{R}^{c} \gamma^{\mu} \ell_{L}$ \\
0 & 0 & 3 & 2 & $7 / 6$ & $\bar{q}_{L} e_{R}$ or $\bar{u}_{R} \ell_{L}$ \\
0 & 0 & 3 & 2 & $1 / 6$ & $\bar{d}_{R} \ell_{L}$ \\
1 & 0 & 3 & 1 & $2 / 3$ & $\bar{q}_{L} \gamma^{\mu} \ell_{L}$ or $\bar{d}_{R} \gamma^{\mu} e_{R}$ \\
1 & 0 & 3 & 1 & $5 / 3$ & $\bar{u}_{R} \gamma^{\mu} e_{R}$ \\
1 & 0 & 3 & 3 & $2 / 3$ & $\bar{q}_{L} \gamma^{\mu} \ell_{L}$ \\
\hline \hline
\end{tabular}

If we do not require leptoquark states to couple directly with SM fermions, different assignments of quantum numbers become possible $[2,3]$.

The Pati-Salam model [4] is an example predicting the existence of a leptoquark state. In this model a vector leptoquark appears at the scale where the Pati-Salam SU(4) "color" gauge group breaks into the familiar $\mathrm{QCD} \mathrm{SU}(3)_{C}$ group (or 
$\left.\mathrm{SU}(3)_{C} \times \mathrm{U}(1)_{B-L}\right)$. The Pati-Salam leptoquark is a weak isosinglet and its hypercharge is $2 / 3$. The coupling strength of the Pati-Salam leptoquark is given by the QCD coupling at the Pati-Salam symmetry breaking scale. Vector leptoquark states also exist in grand unification theories based on $\mathrm{SU}(5)$ [5], $\mathrm{SO}(10)$ [6] which includes Pati-Salam color SU(4), and larger gauge groups. Scalar quarks in supersymmetric models with R-parity violation may also have leptoquark-type Yukawa couplings. The bounds on the leptoquark states can therefore be applied to constraining R-parity violating supersymmetric models. Scalar leptoquarks are expected to exist at $\mathrm{TeV}$ scale in extended technicolor models $[7,8]$, where leptoquark states appear as the bound states of techni-fermions. Compositeness of quarks and leptons also provides examples of models which may have light leptoquark states [9].

Bounds on leptoquark states are obtained both directly and indirectly. Direct limits are from their production cross sections at colliders, while indirect limits are calculated from the bounds on the leptoquark-induced four-fermion interactions which are obtained from low energy experiments.

If a leptoquark couples to fermions of more than a single generation in the mass eigenbasis of the SM fermions, it can induce four-fermion interactions causing flavor-changing-neutralcurrents and lepton-family-number violations. Non-chiral leptoquarks, which couple simultaneously to both left- and righthanded quarks, cause four-fermion interactions affecting the $(\pi \rightarrow e \nu) /(\pi \rightarrow \mu \nu)$ ratio [10]. Non-chiral scalar leptoquark also contributes to the muon anomalous magnetic moment [11,12]. Indirect limits provide stringent constraints on these leptoquarks. Since the Pati-Salam leptoquark has non-chiral coupling with both $e$ and $\mu$, indirect limits from the bounds on $K_{L} \rightarrow \mu e$ lead to a severe bound on the Pati-Salam leptoquark mass.

It is therefore often assumed that a leptoquark state couples only to a single generation in a chiral interaction, where indirect limits become much weaker. This assumption gives strong constraints on concrete models of leptoquarks, however. Leptoquark states which couple only to left- or 
right-handed quarks are called chiral leptoquarks. Leptoquark states which couple only to the first (second, third) generation are referred to as the first (second, third) generation leptoquarks. Davidson, Bailey and Campbell [13] and Leuler [14] give extensive lists of the bounds on the leptoquark induced four-fermion interactions. For the isoscalar scalar and vector leptoquarks $S_{0}$ and $V_{0}$, for example, which couple with the first (second) generation left-handed quark and the first generation left-handed lepton, the bounds of Ref. [13] read $\lambda^{2}<0.03 \times\left(M_{\mathrm{LQ}} / 300 \mathrm{GeV}\right)^{2}$ for $S_{0}$, and $\lambda^{2}<$ $0.02 \times\left(M_{\mathrm{LQ}} / 300 \mathrm{GeV}\right)^{2}$ for $V_{0}\left(\lambda^{2}<5 \times\left(M_{\mathrm{LQ}} / 300 \mathrm{GeV}\right)^{2}\right.$ for $S_{0}$, and $\lambda^{2}<3 \times\left(M_{\mathrm{LQ}} / 300 \mathrm{GeV}\right)^{2}$ for $\left.V_{0}\right)$. The LEP experiments are sensitive to the indirect effects coming from $t$ - and $u$-channel exchanges of leptoquarks in the $e^{+} e^{-} \rightarrow q \bar{q}$ process. The HERA experiments give bounds on the leptoquark induced four-fermion interaction. For detailed bounds obtained in this way, see the Boson Particle Listings for "Indirect Limits for Leptoquarks" and its references.

Collider experiments provide direct limits on the leptoquark states through their pair- and single-production cross sections.

The Tevatron and LEP experiments search for pair- production of the leptoquark states which arises from the leptoquark gauge interaction. The gauge couplings of a scalar leptoquark are determined uniquely according to its quantum numbers in Table 1. Since all of the leptoquark states belong to color triplet representation, the scalar leptoquark pair-production cross section at the Tevatron can be determined solely as a function of the leptoquark mass without making further assumptions. For the first and second generation scalar leptoquark states with decay branching fraction $B(e q)=1$ and $B(\mu q)=1$, the CDF and D0 experiments obtain the lower bounds on the leptoquark mass $>235 \mathrm{GeV}$ (first generation, CDF), > 256GeV (first generation, D0), $>224 \mathrm{GeV}$ (second generation, $\mathrm{CDF}$ ) and $>251 \mathrm{GeV}$ (second generation, D0) at 95\%CL [15]. On the other hand, the magnetic-dipole-type and the electric-quadrupole-type interactions of a vector leptoquark are not determined even if we fix its gauge quantum numbers as listed in the table [16]. We need 
extra assumptions about these interactions to evaluate the pair production cross section for a vector leptoquark.

The searches for the leptoquark single-production are performed by the HERA experiments. Since the leptoquark singleproduction cross section depends on the leptoquark Yukawa coupling, the leptoquark limits from HERA are usually displayed in the mass-coupling plane. For leptoquark Yukawa coupling $\lambda=0.1$, the ZEUS bounds on the first generation leptoquarks range from 248 to $290 \mathrm{GeV}$ depending on the leptoquark species [17]. Similar bounds are obtained by H1 [18]. The LEP experiments also search for the single-production of leptoquark states from the process $e \gamma \rightarrow L Q+q$.

\section{Reference}

1. W. Buchmüller, R. Rückl, and D. Wyler, Phys. Lett. B191, 442 (1987).

2. K.S. Babu, C.F. Kolda, and J. March-Russell, Phys. Lett. B408, 261 (1997).

3. J.L. Hewett and T.G. Rizzo, Phys. Rev. D58, 055005 (1998).

4. J.C. Pati and A. Salam, Phys. Rev. D10, 275 (1974).

5. H. Georgi and S.L. Glashow, Phys. Rev. Lett. 32, 438 (1974).

6. H. Georgi, AIP Conf. Proc. 23, 575 (1975); H. Fritzsch and P. Minkowski, Ann. Phys. 93, 193 (1975).

7. For a review, see, E. Farhi and L. Susskind, Phys. Reports 74, 277 (1981).

8. K. Lane and M. Ramana, Phys. Rev. D44, 2678 (1991).

9. See, for example, B. Schrempp and F. Schrempp, Phys. Lett. B153, 101 (1985).

10. O. Shanker, Nucl. Phys. B204, 375 (1982).

11. U. Mahanta, Eur. Phys. J. C21, 171 (2001) [Phys. Lett. B515, 111 (2001)].

12. K. Cheung, Phys. Rev. D64, 033001 (2001).

13. S. Davidson, D. C. Bailey and B. A. Campbell, Z. Phys. C61, 613 (1994).

14. M. Leurer, Phys. Rev. D49, 333 (1994); Phys. Rev. D50, 536 (1994).

15. G. Chiarelli, arXiv:hep-ex/0509037.

16. J. Blümlein, E. Boos, and A. Kryukov, Z. Phys. C76, 137 (1997). 
17. S. Chekanov et al. [ZEUS Collaboration], Phys. Rev. D68, 052004 (2003).

18. C. Adloff et al. [H1 Collaboration], Phys. Lett. B523, 234 (2001). 